\title{
GRB 040403: A faint X-ray rich gamma-ray burst discovered by INTEGRAL^
}

\author{
S. Mereghetti ${ }^{1}$, D. Götz ${ }^{1,2}$, M. I. Andersen ${ }^{3}$, A. Castro-Tirado ${ }^{4}$, F. Frontera ${ }^{5,6}$, J. Gorosabel ${ }^{4}$, D. H. Hartmann ${ }^{7}$, \\ J. Hjorth ${ }^{8}$, R. Hudec ${ }^{9}$, K. Hurley ${ }^{10}$, G. Pizzichini ${ }^{6}$, N. Produit ${ }^{11}$, A. Tarana ${ }^{12}$, M. Topinka ${ }^{9}$, \\ P. Ubertini ${ }^{12}$, and A. de Ugarte $^{4}$ \\ 1 Istituto di Astrofisica Spaziale e Fisica Cosmica - CNR, Sezione di Milano “G.Occhialini”, via Bassini 15, \\ 20133 Milano, Italy \\ e-mail: sandro@mi.iasf.cnr.it \\ 2 Dipartimento di Fisica, Università degli Studi di Milano Bicocca, P.zza della Scienza 3, 20126 Milano, Italy \\ 3 Astrophysikalisches Institut Potsdam, An der Sternwarte 16, 14482 Potsdam, Germany \\ ${ }^{4}$ Instituto de Astrofísica de Andalucía (IAA-CSIC), Apartado de Correos 3004, 18080 Granada, Spain \\ 5 Physics Department, University of Ferrara, via Paradiso 12, 44100 Ferrara, Italy \\ ${ }^{6}$ Istituto di Astrofisica Spaziale e Fisica Cosmica - CNR, Sezione di Bologna, via Gobetti 101, 40129 Bologna, Italy \\ 7 Clemson University, Department of Physics \& Astronomy, Clemson, SC 29634-0978, USA \\ 8 Niels Bohr Institute, Astronomical Observatory, University of Copenhagen, Juliane Maries Vej 30, 2100 Copenhagen, \\ Denmark \\ 9 Astronomical Institute, Academy of Sciences of the Czech Republic, 25165 Ondrejov, Czech Republic \\ ${ }^{10}$ University of California at Berkeley, Space Sciences Laboratories, Berkeley, CA 94720-7450, USA \\ 11 Integral Science Data Centre, Chemin d'Écogia 16, 1290 Versoix, Switzerland \\ 12 Istituto di Astrofisica Spaziale e Fisica Cosmica - CNR, Sezione di Roma, via Fosso del Cavaliere 100, 00133 Roma, Italy
}

Received 20 September 2004 / Accepted 1 December 2004

\begin{abstract}
GRB 040403 is one of the faintest gamma-ray bursts for which a rapid and accurate localization has been obtained. Here we report on the gamma-ray properties of this burst, based on observations with the IBIS instrument aboard INTEGRAL, and the results of searches for its optical afterglow. The steep spectrum (power law photon index $=1.9$ in the 20-200 keV range) implies that GRB 040403 is most likely an X-ray rich burst. Our optical limit of $R>24.2$ at $16.5 \mathrm{~h}$ after the burst, indicates a rather faint afterglow, similar to those seen in other relatively soft and faint bursts.
\end{abstract}

Key words. gamma rays: bursts - gamma rays: observations

\section{Introduction}

Gamma-ray bursts (GRBs) are extremely energetic cosmic explosions associated with the death of massive stars (see, e.g., Hurley et al. 2002, for a recent review). Multi-waveband observations, made possible by accurate (arc minutes) and fast (minutes) localizations of the rapidly fading gamma-ray emission, are crucial for the development of an understanding of this phenomenon. Rapid follow-up observations also provide a unique opportunity to probe the early universe, as typical GRB redshifts are of order unity. Currently, fast localizations are obtained for a few dozen GRBs per year, based on detections with HETE II and INTEGRAL (Lamb et al. 2003;

* Based on observations with INTEGRAL, an ESA project with instruments and science data centre funded by ESA member states (especially the PI countries: Denmark, France, Germany, Italy, Switzerland, Spain), Czech Republic and Poland, and with the participation of Russia and the USA.
Mereghetti et al. 2004). A significant increase in this rate is expected with the imminent launch of the Swift satellite (Gehrels et al. 2004). It has been pointed out that, thanks to the high sensitivity of its IBIS instrument (Ubertini et al. 2003), the sample of INTEGRAL GRBs is likely to contain a large fraction of bursts at high redshift (Gorosabel et al. 2004). In this respect, the faintest and spectrally softest bursts are the most promising cosmological probes.

On 2004 April 3, a faint GRB triggered the INTEGRAL Burst Alert System (IBAS, Mereghetti et al. 2003a). An IBAS Alert Packet containing the burst coordinates with an uncertainty of only $2.8^{\prime}$ was automatically distributed at 05:08:29 UT. The first photons from the burst had reached the INTEGRAL satellite only $30 \mathrm{~s}$ earlier. This is therefore one of the most precise and rapid GRB localizations obtained to date. A quick look analysis of the data confirmed the automatically derived position and indicated some evidence for a relatively soft spectrum (Götz et al. 2004). A refined analysis, 
announced within three hours of the GRB event, reduced the positional uncertainty to $2.1^{\prime}$. Despite being at a Galactic latitude $\left(b=30^{\circ}\right)$ higher than the majority of INTEGRAL bursts (Mereghetti et al. 2004) and thus barely affected by interstellar extinction $\left(A_{\mathrm{V}} \sim 0.3\right)$, optical follow-ups of GRB 040403 were somewhat discouraged by the presence of a full moon.

Here we present a detailed analysis of the INTEGRAL data and the results of searches for the optical afterglow carried out at the Observatory of Sierra Nevada (OSN) and at the Nordic Optical Telescope (NOT). We also report on pre-GRB images obtained with the Burst Alert Robotic Telescope (BART) at the Ondrejov Observatory, which allow us to place constraints on the presence of optically variable sources before the onset of the prompt GRB emission. To our knowledge, GRB 040403 was not detected by other satellites ${ }^{1}$, and no follow up observations were performed in the $\mathrm{X}$-ray and radio ranges.

\section{INTEGRAL observations}

The data presented here were obtained with ISGRI (Lebrun et al. 2003), the lower energy detector of the IBIS imaging instrument. Due to its $15 \mathrm{keV}-1 \mathrm{MeV}$ energy range, large field of view, high time resolution and good spatial resolution, ISGRI provides the best data used by IBAS for real time search and localization of GRBs.

GRB 040403 occurred at off-axis angles of $Z=-4.28^{\circ}$ and $Y=-4.34^{\circ}$, in the fully coded region of the instrument field of view ${ }^{2}$. The top panels of Fig. 1 show the light curves in the soft (15-40 keV) and hard (40-200 keV) ranges. The burst profile exhibits a single peak with a rise time of about $5 \mathrm{~s}$ and a slower decay. The $T_{90}$ duration is $19 \mathrm{~s}$. The decay time scale is longer in the softer energy band, indicating the presence of spectral hard-to-soft evolution, as observed in many GRBs. This is demonstrated by the hardness ratio evolution (third panel of Fig. 1) and by the time resolved spectral analysis described below. We computed the cross correlation between the light curves at energies below and above $40 \mathrm{keV}$, and found that the soft light curve has a time lag of $0.6 \pm 0.1 \mathrm{~s}$.

In order to measure the time integrated spectrum and the fluence we extracted the counts in a $30 \mathrm{~s}$ long time interval starting at 5:08:00 UT. This yielded about 2500 net counts. For the spectral analysis we used the most recent response matrix, which takes into account the effects of the off-axis angle dependence of the mask transparency. The spectrum was rebinned in order to have at least 20 counts per bin and fitted over the 20-200 keV range. A good fit was obtained with a power law of photon index $\Gamma=1.90 \pm 0.15$ and $20-200 \mathrm{keV}$ flux of 0.2 photons $\mathrm{cm}^{-2} \mathrm{~s}^{-1}\left(\chi^{2} /\right.$ d.o.f. $=12.39 / 12$, see Fig. 2$)$. The fluence in the same energy range was $5.0 \times 10^{-7} \mathrm{erg} \mathrm{cm}^{-2}$ and the peak flux $\sim 0.50$ photons $\mathrm{cm}^{-2} \mathrm{~s}^{-1}$ (over a $1 \mathrm{~s}$ time interval). Time resolved spectral analysis yields the power law

\footnotetext{
1 Two other bursts detected on the same day by other satellites have been erroneously named in Hurley et al. 2004: they occurred after the INTEGRAL burst reported here, and should therefore be named GRB 040403B and GRB 040403C.

${ }^{2}$ IBIS is a coded mask telescope. Sources outside the fully coded field of view project an aperture-modulated flux only on a fraction of the detection plane.
}

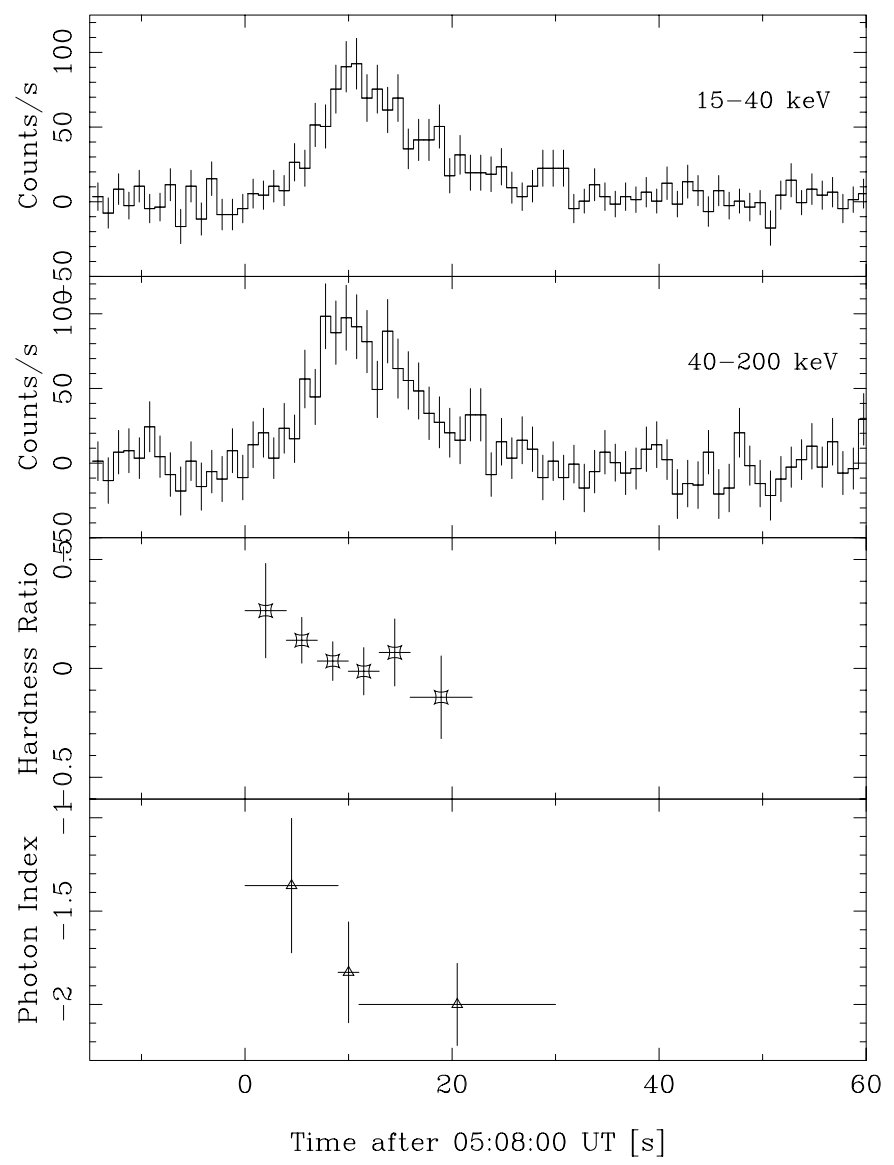

Fig. 1. Light curves and spectral evolution of GRB 040403: a) light curve obtained with IBIS/ISGRI in the 15-40 keV energy range, binned in intervals of $1 \mathrm{~s}$ (only detector elements illuminated by more than $50 \%$ by the source were used); b) same as a) for the $40-200 \mathrm{keV}$ range; c) ratio of $15-40 \mathrm{keV}$ to $40-200 \mathrm{keV}$ counts; and d) the power law photon index obtained from the spectral analysis of three distinct time intervals.

photon indexes shown in the lowest panel of Fig. 1, confirming the hard-to-soft spectral evolution derived from the hardness ratio.

An acceptable fit to the time averaged spectrum could also be obtained with a Band function (Band et al. 1993), which has two more free parameters than the simple power law adopted above. However, owing to the limited statistics and reduced energy range the fit parameters cannot be constrained. The best fit is found for $\alpha \sim-1.5, E_{\mathrm{p}} \sim 60 \mathrm{keV}$, and $\beta>-3$.

We also recomputed the GRB position by producing images in different energy ranges and time intervals. The highest signal to noise ratio was obtained in the $15-100 \mathrm{keV}$ range and the corresponding position of GRB 040403 is $\alpha_{\mathrm{J} 2000}=$ $7^{\mathrm{h}} 40^{\mathrm{m}} 54^{\mathrm{s}}, \delta_{\mathrm{J} 2000}=+68^{\circ} 12^{\prime} 55^{\prime \prime}$. This position, based on the final attitude reconstruction derived for the satellite, is consistent with and supersedes the refined position reported less than three hours after the GRB event (Götz et al. 2004). The $90 \%$ confidence level error radius for the GRB position is $2^{\prime}$. GRB 040403 was not detected above $120 \mathrm{keV}$, consistent with the soft spectrum discussed above. 
GRB 040403

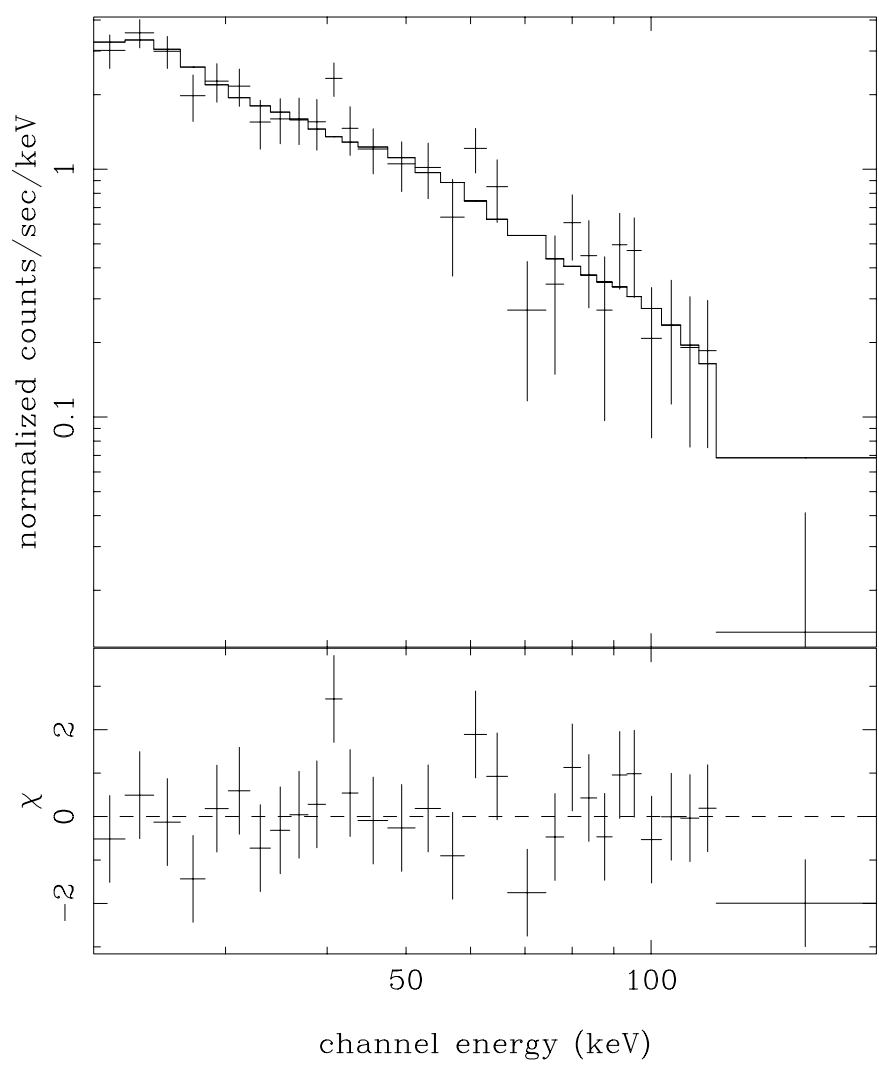

Fig. 2. IBIS/ISGRI spectrum of GRB 040403 fitted with a power law. Upper panel: data and best fit model. Lower panel: residuals from the best fit spectrum in units of sigma.

\section{Optical observations}

\subsection{Pre-burst imaging}

The robotic telescope BART (Jelinek et al. 2003) observed the position of GRB 040403 on April 3, 2004 with two wide-field cameras. Images of the GRB position were obtained at 03:17:32 and 03:36:01 UT (mid-points of 120 s long exposures) i.e. 112 and 93 min before the burst. No variable optical object was detected within the GRB error box. Relatively poor weather conditions limit our ability to reach very faint magnitudes. The magnitude constraints on any pre-GRB emission obtained for these images are $R>12$ and $I>11.6$. The second image was the last usable observation before images started to get overexposed due to the rising sun.

\subsection{Search for optical afterglow emission}

Following the IBAS trigger, we observed the field of GRB 040403 at optical wavelengths starting on April 3.83 UT, with the $1.5 \mathrm{~m}$ telescope at the OSN in Granada and with the $2.5 \mathrm{~m}$ NOT telescope in La Palma. The OSN images in the $R$ band $(6 \times 600 \mathrm{~s})$ were obtained with the CCD VersArray 2048B which provides a $7^{\prime} \times 7^{\prime}$ field of view. Comparison images were acquired on 4 and 20 April 2004 $(9 \times 600 \mathrm{~s})$.

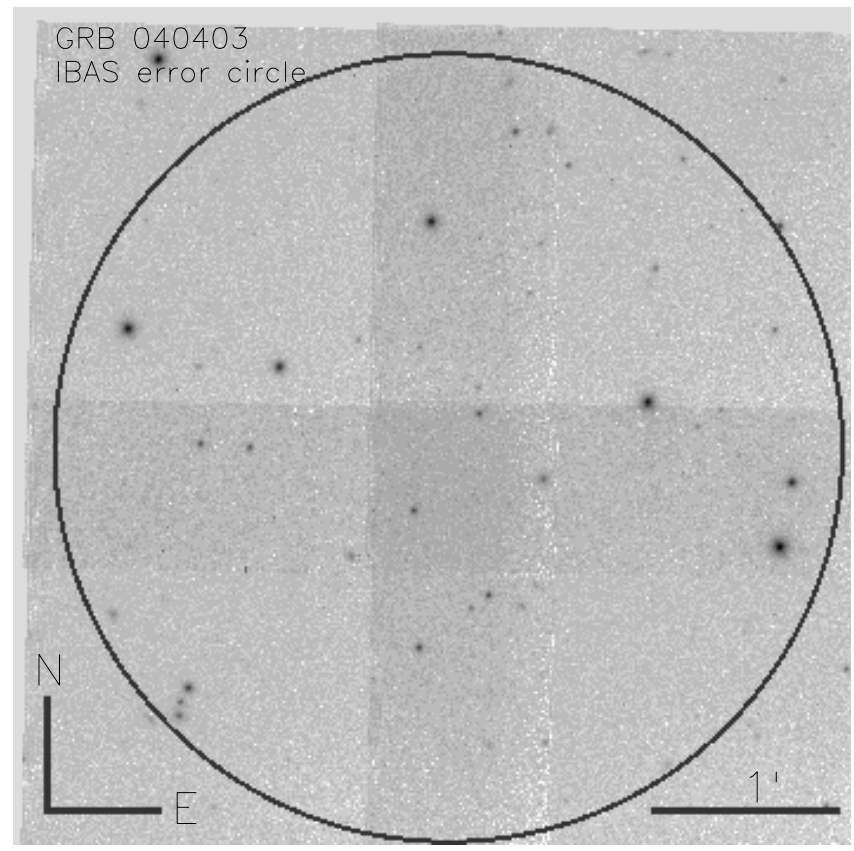

Fig. 3. The $R$-band image of the GRB 040403 error box, obtained with the Nordic Optical Telescope (NOT) on April 4, $17 \mathrm{~h}$ after the GRB. The $2 \times 2$ mosaic image covers the entire 2.1' IBAS error circle. The $3 \sigma$ limiting magnitude for this field is $R_{\lim }=24.2$.

The NOT observations were obtained with the Standby camera (StanCam). As the field of view of StanCam is only 3 arcmin square, a $2 \times 2$ mosaic was acquired, in order to cover the entire error-box, each mosaic element consisting of three integrations of $300 \mathrm{~s}$. The observations were initiated on April 3.890 UT, with a mean epoch of April 3.940 UT. The seeing was $\sim 0.80^{\prime \prime}$ resulting in a $3 \sigma$ limiting magnitude of $R=24.2$. During the following night, second epoch imaging was carried out, but due to poor seeing and partial cloud cover, it was not nearly as deep as the images from the previous epoch. Visual comparison did not reveal any variable object. Subsequent NOT observations, during a possible SN rebrightening phase 10 days after the GRB, were planned but not conducted, due to enduring bad weather. To fully exploit the depth of the first epoch observations, a deeper $R$-band image was acquired on June 21, 2004, using ALFOSC at the NOT, which covers the entire error box in a single exposure. The resulting image, which was composed of five $300 \mathrm{~s}$ exposures, obtained under seeing of $0.85^{\prime \prime}$, reaches half a magnitude deeper than the first epoch images. Visual comparison of the residual image after image subtraction does not reveal any variable source to the detection limit of the first epoch. The position dependent variable PSF of the ALFOSC images effectively prevents quantitative image subtraction from being employed on this data set. We decided not to combine the OSN and the NOT data, as the difference in depth and seeing is too large to allow a significantly lower limit from combined data. Several galaxies fainter than $R \sim 20$ contained in the INTEGRAL error region could be considered as the potential GRB host, but, lacking a more precise position, none can be singled out. 


\section{Discussion}

GRB 040403 is one of the faintest GRBs detected by INTEGRAL to date. The soft spectrum measured with ISGRI indicates that it likely belongs to the class of X-ray rich GRBs. The distinction between normal GRBs, X-ray rich GRBs and $\mathrm{X}$-ray Flashes (XRF) is somewhat arbitrary, but a widely used definition is based on the ratio of the fluences in the 2-30 and $30-400 \mathrm{keV}$ energy ranges, $S=\log \left(F_{2-30} / F_{30-400}\right)$. XRFs have $S>0$, normal GRBs have $S<-0.5$, and bursts with intermediate values are considered X-ray rich (Lamb et al. 2003). We do not have information on the GRB 040403 spectrum below $15 \mathrm{keV}^{3}$ and we have only ISGRI upper limits above $\sim 150 \mathrm{keV}$. Assuming that the GRB spectrum is well represented by a single power law over the entire relevant range, we infer $S=$ $-0.11 \pm 0.18$. Only a significant spectral flattening to a photon index of $\sim 0.5$ below $\sim 20-30 \mathrm{keV}$ would result in values of $S$ closer to those observed in typical GRBs. Also adopting Band spectra with various parameter values in the range allowed by our spectral fits, we obtain values of $S$ larger than -0.5 . We thus conclude that GRB 0040403 was most likely a member of the class of X-ray rich GRBs.

Our afterglow limit of $R>24.2$ at $16.5 \mathrm{~h}$ after the burst and those reported soon after the event $(R \geq 19$ at $1.1 \mathrm{~h}$; Moran et al. 2004) are rather constraining, compared to the majority of limits obtained to date for other bursts. Most of the observed GRB afterglows are brighter than $R \sim 23$ at $t=1$ day (Fynbo et al. 2001; Fox et al. 2003; Berger et al. 2002). It is interesting to note that a similarly faint optical afterglow was reported also for GRB 030227, another faint and spectrally soft burst discovered by INTEGRAL (Mereghetti et al. 2003b; Castro-Tirado et al. 2003).

High redshift could be responsible for the faintness of the optical afterglows and the X-ray richness of these faint INTEGRAL bursts. Based on the correlation between spectral lag and luminosity established for GRBs (Norris et al. 2000), the $0.6 \mathrm{~s}$ lag measured in GRB 040403 corresponds to an isotropic luminosity of $\sim 1.2 \times 10^{51} \mathrm{erg} \mathrm{s}^{-1}$. For such a luminosity, the flux measured by INTEGRAL implies a redshift $z=2.1^{4}$. More extensive prompt multi-wavelength followup observations of the IBAS Alerts for faint bursts are clearly needed to assess whether INTEGRAL is indeed preferentially sampling the farthest GRBs.

We finally note that the upper limit from the image obtained on April 20 also excludes the presence of a nearby $(z=0.1-0.2)$ underlying, non-obscured supernova similar to SN 1998bw (Galama et al. 1998) in the error box. It is now fairly well established that most long-duration GRBs are followed by extra light, commonly attributed to an associated supernova (Zeh et al. 2004). This link is a natural consequence of the collapsar model (Woosley 1993), and GRB 030329 is so far the best direct proof (Stanek et al. 2003; Hjorth et al. 2003; Greiner et al. 2003) of this supernova-GRB association. HETE-2 has provided strong evidence that X-ray flashes (XRFs), X-ray rich

\footnotetext{
3 The burst was outside the field of view of the JEM-X instrument (4-35 keV, Lund et al. 2003) and of the optical camera (OMC, Mas Hesse et al. 2003) aboard INTEGRAL.

${ }^{4}$ We have assumed $H_{0}=65 \mathrm{~km} \mathrm{~s}^{-1} \mathrm{Mpc}^{-1}, \Omega_{\Lambda}=0.7$ and $\Omega_{\mathrm{m}}=0.3$.
}

GRBs, and GRBs are in fact drawn from the same underlying source population (Lamb et al. 2004). It is thus natural to assume that X-ray rich GRBs are also followed by emission from a supernova. The lack of deep late time observations for this GRB unfortunately does not allow us to test this hypothesis, but we are very optimistic that future rapid GRB localizations with INTEGRAL, HETE-2, or Swift will eventually reveal enough to decide whether or not the "XRF-GRB continuum" hypothesis holds.

Acknowledgements. This research has been partially supported by the Italian Space Agency. K.H. and D.H. are grateful for support under NASA Grant NAG5-12706 in the INTEGRAL US Guest Investigator program.

\section{References}

Band, D. L., Matteson, J., Ford, L., et al. 1993, ApJ, 413, 281

Berger, E., Kulkarni, S. R., Bloom, J. S., et al. 2002, ApJ, 581, 981

Castro-Tirado, A. J., Gorosabel, J., Guziy, S., et al. 2003, A\&A, 411, L315

Fynbo, J. U., Jensen, B. L., Gorosabel, J., et al. 2001, A\&A, 369, 373 Fox, D. W., Price, P. A., Soderberg, A. M., et al. 2003, ApJ, 586, L5

Galama, T. J., Vreeswijk, P. M., van Paradijs, J., et al. 1998, Nature, 395,670

Gehrels, N., Chincarini, G., Giommi, P., et al. 2004, ApJ, 611, 1005

Gorosabel, J., Lund, N., Brandt, S., Westergaard, N. J., \& Castro Ceron, J. M. 2004, A\&A, 427, 87

Götz, D., Mereghetti, S., Borkowski, J., \& Beck, M. 2004, GCN, 2560

Greiner, J., Klose, S., Reinsch, K., et al. 2003, Nature, 426, 157

Hjorth, J., Sollerman, J., Møller, P., et al. 2003, Nature, 423, 847

Hurley, K., Sari, R., \& Djorgovski, S. G. 2002 [arXiv: astro-ph/0211620]

Hurley, K., et al. 2004, GCN Circ., No. 2566

Jelinek, M., et al. 2003, in Gamma-ray burst and aferglow astronomy 2001: A Workshop Celebrating the First Year of the HETE Mission, AIP Conf. Proc., 662, 520

Lamb, D. Q., Ricker, G. R., Atteia, J.-L., et al. 2003, New Astron. Rev., 48, 423

Lamb, D. Q., Donaghy, T. Q., \& Graziani, C. 2004, New Astr. Rev., 48,459

Lebrun, F., Leray, J. P., Lavocat, P., et al. 2003, A\&A, 411, L141

Lund, N., Budtz-Jrgensen, C., Westergaard, N. J., et al. 2003, A\&A, 411, L131

Mas Hesse, J. M., Gimenez, A., Culhane, J. L., et al. 2003, A\&A, 411, L261

Mereghetti, S., Götz, D., Borkowski, J., et al. 2004, Proc. 5th INTEGRAL Workshop, The INTEGRAL Universe, February 16-20, 2004 [arXiv: astro-ph/0404019]

Mereghetti, S., Götz, D., Borkowski, J., Walter, R., \& Pedersen, H. 2003a, A\&A, 411, L291

Mereghetti, S., Götz, D., Tiengo, A., et al. 2003b, ApJ, 590, L73

Moran, J., Nysewander, M., \& Reichart, D. 2004, GCN Circ., No. 2561

Norris, J. P., Marani, G. F., \& Bonnell, J. T. 2000, ApJ, 534, 248

Stanek, K. Z., Matheson, T., Garnavich, P. M., et al. 2003, ApJ, 591, L17

Ubertini, P., Lebrun, F., Di Cocco, G., et al. 2003, A\&A, 411, L131

Woosley, S. E. 1993, ApJ, 405, 273

Zeh, A., Klose, S., \& Hartmann, D. H. 2004, ApJ, 609, 952 